\title{
The substrates of Plk1, beyond the functions in mitosis
}

\author{
X. Shawn Liu ${ }^{1 *}$, Bing Song ${ }^{2 *}$, Xiaoqi Liu ${ }^{1,3 凶}$ \\ ${ }^{1}$ Department of Biochemistry, Purdue University, West Lafayette, Indiana 47907, USA \\ 2 Department of Biological Sciences, Purdue University, West Lafayette, Indiana 47907, USA \\ ${ }^{3}$ Center for Cancer Research, Purdue University, West Lafayette, Indiana 47907, USA \\ $\triangle$ Correspondence: liu8@purdue.edu \\ Received November 3, 2010 Accepted November 8, 2010
}

\begin{abstract}
Polo-like kinase 1 (Plk1) is a key regulator of cell division in eukaryotic cells. In this short review, we briefly summarized the well-established functions modulated by Plk1 during mitosis. Beyond mitosis, we focused mainly on the unexpected processes in which PIk1 emerges as a critical player, including microtubule dynamics, DNA replication, chromosome dynamics, p53 regulation, and recovery from the G2 DNA-damage checkpoint. Our discussion is mainly based on the critical substrates targeted by Plk1 during these cellular events and the functional significance associated with each phosphorylation event.
\end{abstract}

KEYWORDS Polo-like kinase 1, phosphorylation, substrates

\section{INTRODUCTION}

It has been 23 years since the polo gene was discovered in Drosophila melanogaster, whose loss-of-function mutations are associated with various mitotic defects (Sunkel and Glover, 1988). This gene encodes a serine/threonine kinase and is highly conserved from yeast to human. Among all family members, including mammalian Plk1/2/3, Xenopus Plx1/2/3, fission yeast Plo1, and budding yeast Cdc5, mammalian Polo-like kinase 1 (Plk1) has been most extensively studied during the past decade. Plk1 contains a canonical serine/threonine kinase domain at the $\mathrm{N}$ terminus and a C-terminal regulatory domain termed polo-box domain (PBD) (Strebhardt, 2010) (Fig. 1). The PBD binds to the Ser[pSer/pThr]-Pro/X motif generated by other priming kinases or
Plk1 itself, and subsequently localizes Plk1 to various cellular structures, such as centrosomes, spindle poles, kinetochores, midzones and midbodies (Elia et al., 2003).

\section{PLK1 IN CANCER BIOLOGY}

A close correlation between mammalian Plk1 expression and carcinogenesis has been documented, and overexpression of Plk1 has been observed in various human tumors (Eckerdt et al., 2005; Takai et al., 2005). For example, elevated Plk1 expression was detected among patients with melanomas, and patients with moderate Plk1 expression survived significantly longer than those with high Plk1 levels (Strebhardt et al., 2000). Similarly, non-small cell lung cancer patients whose tumors showed moderate Plk1 expression survived significantly longer than patients with high levels of Plk1 transcripts (Wolf et al., 1997). Analysis of patients with head and neck carcinomas, and esophageal and gastric cancer also revealed a close correlation between lower survival rates and higher Plk1 expression levels (Knecht et al., 1999; Knecht et al., 2000; Takai et al., 2005). Therefore, it was proposed that Plk1 could serve as a novel diagnostic marker for several types of cancers (Eckerdt et al., 2005; Takai et al., 2005). Moreover, constitutive expression of Plk1 in NIH3T3 cells caused oncogenic focus formation and induced tumor growth in nude mice (Smith et al., 1997). Thus, inhibition of Plk1 function may be an important approach for cancer therapy. In line with this notion, it was shown that injection of plasmids containing shRNA to target Plk1 inhibited tumor growth in nude mice (Spänkuch et al., 2004). Significantly, several Plk1 inhibitors, such as BI2536 and GSK461364, are in phase I or II clinical studies for patients with various cancers (Strebhardt, 2010).

"These authors contributed equally to the work. 


\section{MAJOR MITOTIC FUNCTIONS OF PLK1}

Enough evidence has established Plk1 as a key player during mitosis. Plk1-mediated phosphorylation event is involved in almost every aspect of mitotic events. For example, during the onset of mitosis, Plk1 phosphorylatation of cyclin B and Cdc25C, two critical regulators of Cdk1, leads to Cdk1 activation for mitotic entry. Plk1 has an essential role in chromosome condensation/separation during mitosis. The initial examination of Polo mutants in Drosophila revealed hypercondensed chromosomes (Llamazares et al., 1991). These observations matched later studies where RNAimediated Plk1 depletion in well-synchronized HeLa cells led to the formation of circular condensed chromosomes (Liu et al., 2005). Plk1 is clearly involved in bipolar spindle formation during mitosis. Several proteins are involved in this process, such as NIP (ninein-like protein), an important component of the centrosome and essential for microtubule nucleation; TCTP, a microtubule-stabilizing protein; and Op18, a microtubule destabilizing protein. All of these proteins are phosphorylated by Plk1, which regulates their functions during spindle formation (Budde et al., 2001; Yarm, 2002; Casenghi et al., 2003). By localizing in kinetochores at prometaphase, the chromosomal structures to which spindle microtubule attach, Plk1 phosphorylates additional substrates, such as BubR1, PBIP1 and PICH, to assist establishment of bipolar spindle (Kang et al., 2006; Baumann et al., 2007; Elowe et al., 2007). These well-established Plk1 substrates have been the subject of many extensive reviews (Strebhardt, 2010). In this manuscript, we only focus on several novel Plk1 substrates identified in our laboratory.
These substrates have diverse cellular functions, including microtubule dynamics, DNA replication, chromosome dynamics, p53 regulation, and recovery from the G2 DNAdamage checkpoint (Fig. 1).

\section{PLK1 IN MICROTUBULE DYNAMICS}

Microtubules have an intrinsic structural polarity and are generally oriented in cells with their minus ends at the centrosome and their plus ends pointing toward the cell periphery during interphase. During mitosis, microtubules must attach paired sister chromatids to opposite poles of the spindle, which is essential to ensure proper distribution of the sister chromatids to the two daughter cells. Microtubule dynamics consist of alternating phases of growth and shortening, a pattern of behavior known as dynamic instability (Mitchison and Kirschner, 1984). This process is tightly regulated by a group of proteins that bind specifically to the plus ends of the growing microtubules (+ TIPs). Clip-170, the founding member of the microtubule plus end family (Perez et al., 1999), is composed of three separate regions: $\mathrm{N}$ terminus, central coiled-coil region, and $\mathrm{C}$ terminus (Fig. 2A). In addition to two conserved cytoskeleton-associated protein glycinerich (CAP-Gly) domains, the $\mathrm{N}$ terminus has three serine-rich regions. The $\mathrm{N}$-terminal domain plays an essential role in microtubule targeting, the long central coiled-coil domain is responsible for dimerization of the protein, and the $\mathrm{C}$ terminus, which contains two zinc finger domains, interferes with microtubule binding by interacting with the $\mathrm{N}$ terminus. Enough evidence has accumulated to support the notion that Clip-170 plays an important role in microtubule dynamics. In
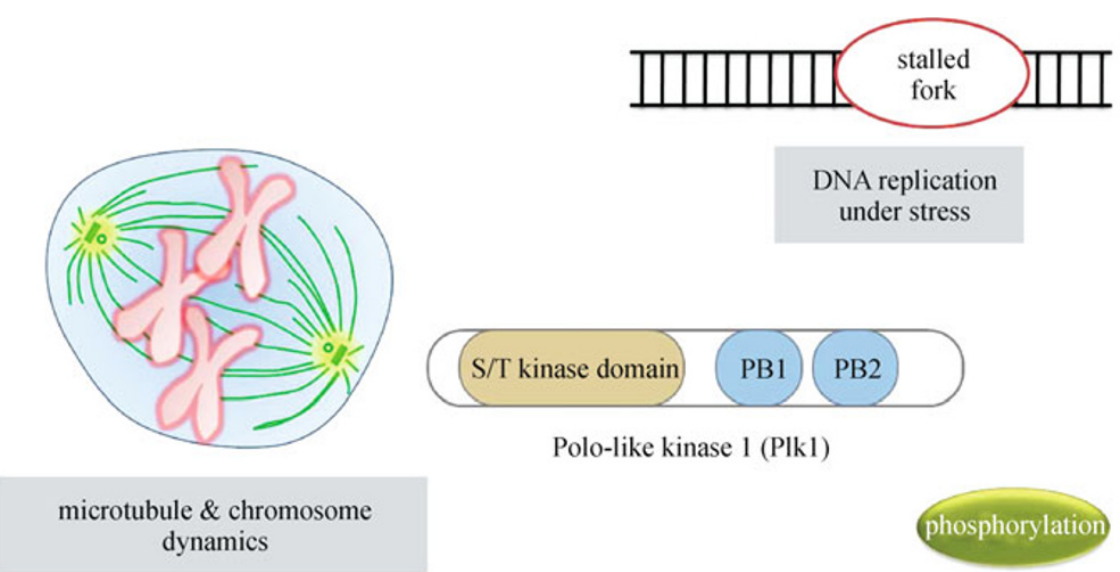

Polo-like kinase 1 (Plk1)

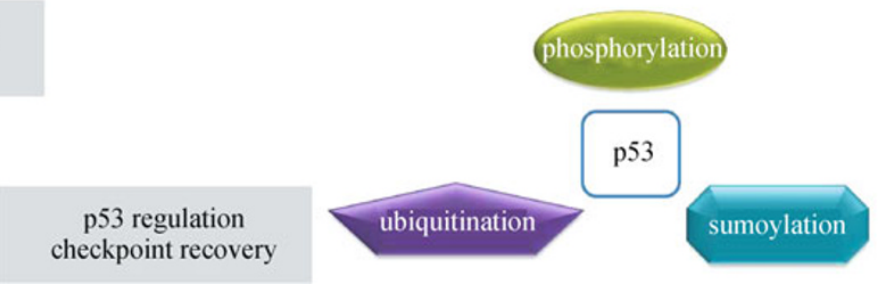

Figure 1. Multiple functions of Polo-like kinase 1. Several recently identified Plk1 substrates reveal that Plk1 has functions beyond mitosis, such as microtubule and chromosome dynamics, DNA replication licensing under a specific cellular context, p53 regulation, and recovery from the G2 DNA-damage checkpoint. 
addition to its positive role in regulating microtubule growth in both yeast and humans (Brunner and Nurse, 2000; Komarova et al., 2002), Clip-170 is involved in recruitment of dynactin to the microtubule plus ends and in linking microtubules to the cortex through Cdc42 and IQGAP (Fukata et al., 2002; Lansbergen et al., 2004). The role of Clip-170 during mitosis was recently examined by loss-of-function approaches. It was shown that Clip-170 localizes to unattached kinetochores in prometaphase, and such localization is essential for the formation of kinetochore-microtubule attachments (Tanenbaum et al., 2006).

p150 glued, a component of the dynein/dynactin complex, and LIS1, two major Clip-170-interacting partners, are localized to both microtubule plus ends and kinetochores. While p $150^{\text {glued }}$ localization at microtubule plus ends is Clip170-dependent (Lansbergen et al., 2004), Clip-170kinetochore localization relies on a functional dynactin complex (Coquelle et al., 2002). Further mapping experiments indicated that the $\mathrm{C}$ terminus of Clip-170 directly interacts with the $\mathrm{N}$ terminus of $\mathrm{p} 150^{\text {glued }}$ (Lansbergen et al., 2004). Binding to Clip-170 in a zinc finger domain-dependent manner, LIS1 directly interacts with dynein/dynactin complex as well (Fig. 2B). While LIS1 recruitment to kinetochores is dynein/dynactin dependent (Coquelle et al., 2002), dominant negative inhibition of LIS1 also leads to displacement of Clip170 from kinetochores (Tai et al., 2002). Therefore, both dynactin and LIS1 are essential for kinetochore localization of Clip-170 during prometaphase. Similar to dynactin, LIS1 localization to microtubule plus ends depends on the $C$ terminus of Clip-170 (Coquelle et al., 2002). Indeed, LIS1 and p150 glued compete for binding to the C-terminal zinc finger domain of Clip-170 (Lansbergen et al., 2004).
Post-translational modification is one major mechanism to regulate protein functions. Both Clip-170 and $\mathrm{p} 150^{\text {glued }}$ are phosphoproteins in vivo and phosphorylation regulates their microtubule binding activities (Rickard and Kreis, 1991; Vaughan et al., 2002). Furthermore, multiple kinases are proposed to be responsible for Clip-170 phosphorylation in vivo (Choi et al., 2002). However, the nature of these kinases is still unknown until recently.

We recently identified Cdk1, Plk1 and CK2 (Casein kinase 2) as three kinases of CLIP-170 (Yang et al., 2009a; Li et al., 2010a). We showed that Cdk1 targets CLIP-170 at Thr287 in vivo. Significantly, expression of CLIP-170 with a threonine 287 to alanine substitution (T287A) results in its mislocalization, accumulation of Plk1 and cyclin B, and block of the G2/M transition. Finally, we found that depletion of CLIP170 leads to centrosome reduplication and that Cdk1 phosphorylation of CLIP-170 is required for the process. These results demonstrated that Cdk1-mediated phosphorylation of CLIP-170 is essential for the normal function of this protein during cell cycle progression (Yang et al., 2009a). We subsequently mapped Ser195 and Ser1318 as phosphorylation sites of Plk1 and CK2, respectively. We showed that a CK2 unphosphorylatable mutant lost its ability to bind to dynactin and to localize to kinetochores during prometaphase, indicating that the CK2 phosphorylation of CLIP-170 is involved in its dynactin-mediated kinetochore localization. Furthermore, we provided evidence that Plk1 phosphorylation of CLIP-170 at S195 enhances its association with CK2. Finally, we detected defects in the formation of kinetochore fibers in cells expressing the CLIP-S195A and -S1318A, but not the CLIP-S195E and -S1318D, confirming that Plk1- and CK2-associated phosphorylation of CLIP-170 is indeed

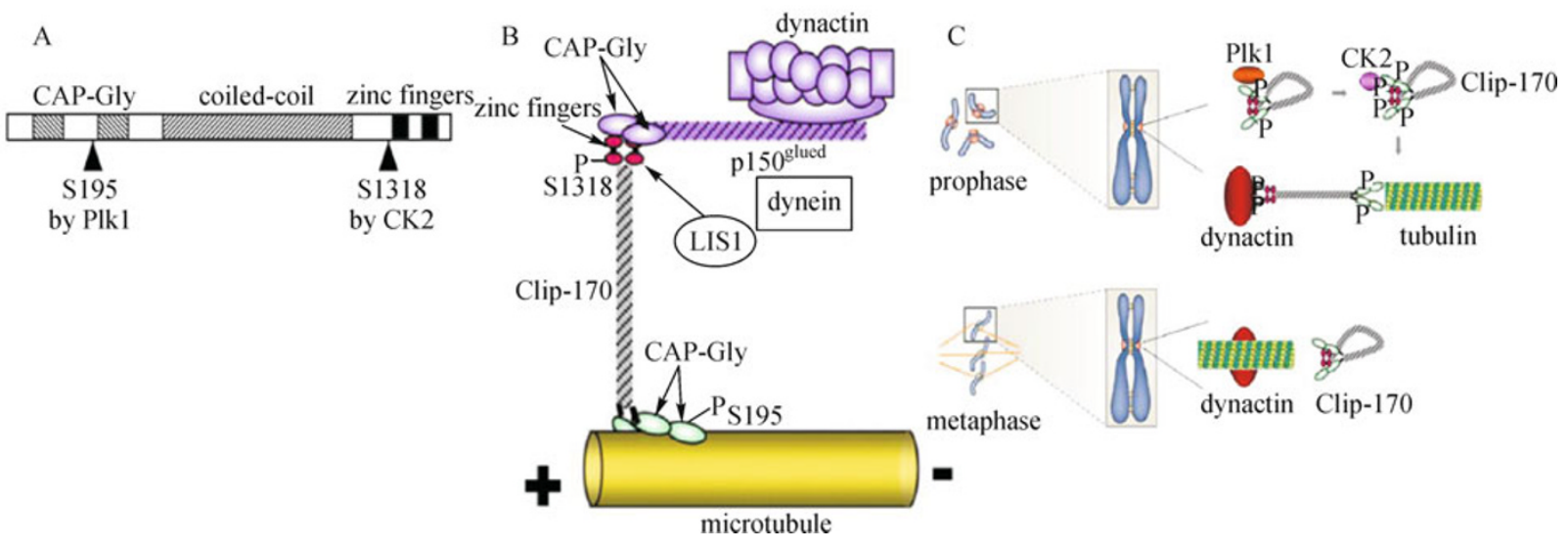

Figure 2. Clip-170 is a novel substrate of PIk1. (A) Schematic representation of Clip-170 domains. S195, located in the Nterminal domain, is targeted by Plk1, whereas S1318, located in the C-terminal domain, is phosphorylated by CK2. (B) The possible interactions between Clip-170, dynactin, dynein, and LIS1 at the plus end of a growing microtubule. It was proposed that the Cterminal zinc-finger domain of Clip-170 interacts with the N-terminal CAP-Gly domain of p150 glued, and the N-terminal CAP-Gly domain of Clip-170 binds to microtubule plus ends. LIS1 interacts with both dynein and Clip-170. (C) We hypothesize that phosphorylation of Clip-170 by both Plk1 and CK2 is essential for the formation of kinetochore-microtubule attachements. Plk1 phosphorylation of Clip-170 at S195 enhances its binding for CK2. CK2 further phosphorylates Clip-170 at S1318, which is essential for its interaction with dynactin, thus facilitating the subsequent kinetochore-microtubule attachments. 
involved in the formation of kinetochore-microtubule attachments in mitosis ( $\mathrm{Li}$ et al., 2010a). These studies provided more detailed mechanisms to understand how CLIP-170 is regulated by post-translation modifications (Fig. 2C).

Furthermore, we also reported $\mathrm{p} 150^{\text {glued }}$, the major component of the dynein/dynactin complex, as a Plk1 substrate during the G2/M transition (Li et al., 2010b). Nuclear envelope breakdown (NEBD) is an essential step during the G2/M transition in higher eukaryotic cells. Increasing evidence supports the notion that both microtubules and microtubule-associated motor proteins are critical regulators of NEBD. Although it has been described that $\mathrm{p} 150^{\text {glued }}$ localizes in the nuclear envelope (NE) during prophase, the exact role of $\mathrm{p} 150^{\text {glued }}$ and its regulation during NEBD are largely elusive. We showed that in mammalian cells both Plk1 and $\mathrm{p} 150^{\text {glued }}$ regulate NEBD and that Plk1 interacts with and phosphoryates $\mathrm{p} 150^{\text {glued }}$ during NEBD at prophase. Using various approaches, we showed that Plk1 phosphorylates p150 ${ }^{\text {glued }}$ at Ser179 and that the pS179 epitope is generated at the NE of prophase cells. Significantly, Plk1-mediated phosphorylation of $\mathrm{p} 150^{\text {glued }}$ at Ser179 positively regulates its accumulation at the NE during prophase. Finally, we found that cells expressing the Plk1-unphosphorylatable mutant

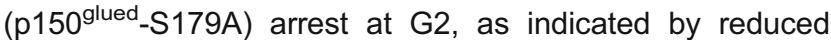
NEBD, increased levels of cyclin B and phospho-H3, but a decreased level of Cdk1 kinase activity. Altogether, we concluded that Plk1 phosphorylation of $\mathrm{p} 150^{\text {glued }}$ might be one major pathway of NEBD regulation.

\section{PLK1 IN DNA REPLICATION}

DNA replication starts with the ordered assembly of a multiprotein complex called the pre-replicative complex (pre-RC), whose components are recruited to origins in a stepwise manner beginning with the origin recognition complex (ORC) (Fig. 3). ORC is a six-subunit complex (Orc1-6) that acts as the initiator, the protein that selects the site for subsequent initiation of replication. It has been shown that the ORC subunits interact with each other and that Orc2 and Orc3 form a core subcomplex with which other ORC members interact (Dhar et al., 2001). The ORC recruits Cdc6 and licensing factor Cdt1, both of which are required for subsequent loading of the Mcm2-7 helicase, which is essential for DNA unwinding prior to replication (Liang et al., 1995). During the G1/S transition, the action of two kinases, Cdc7 and Cdk2/cyclin E, triggers loading of $\mathrm{Cdc} 45$, a protein that is required for both activation of $\mathrm{Mcm}$ on origins and chromosome unwinding at the replication forks (Pacek et al., 2006). Of note, formation of the pre-RC occurs during late $\mathrm{M}$ phase right after sister chromatid segregation and licenses the DNA for next round of replication during the subsequent $S$ phase (Takeda and Dutta, 2005). In other words, DNA replication is intimately coupled with mitotic events.

Increasing evidence suggests that Plk1 is involved in Sphase events (Tsou et al., 2009; Yim and Erikson, 2009). For example, Yim and Erikson used the RNAi approach to demonstrate that Plk1 is required for DNA replication during cell cycle progression (Yim and Erikson, 2009). They showed that Plk1 depletion results in disrupted pre-RC formation and reduced DNA synthesis of the first cell cycle after depletion, likely due to increased levels of Geminin and Emi1, an inhibitor of anaphase-promoting complex. Moreover, Plk1 depletion leads to DNA damage at the first $S$ phase before cells display severe mitotic catastrophe or apoptosis (Yim and Erikson, 2009). Another recent finding also supports the Sphase function of Plk1 (Tsou et al., 2009). DNA replication is coupled with centrosome duplication, another important cellular event, to ensure equal segregation of chromosomes in mitosis. It was shown that Plk1 and separase act during mitosis to license duplication of centrosomes in the following cell cycle (Tsou et al., 2009). Additional evidence to suggest a role of Plk1 in DNA replication includes interaction of Plk1 with pre-RC proteins and co-localization of Plk1 with pre-RC components in centrosomes (Tsvetkov and Stern, 2005; Stuermer et al., 2007). Despite such progress in the field, the question how Plk1-associated kinase activity regulates mitotic licensing of DNA replication in the next round of $S$ phase remains unanswered.

It is widely accepted that replication initiation is regulated by epigenetic phenomena. For example, tethering histone acetyltransferase (HAT) and histone deacetylase to origins can increase and decrease origin activity, respectively (Aggarwal and Calvi, 2004). The best candidate for the HAT working at origins is $\mathrm{Hbo} 1$ (histone acetyltransferase binding to Orc1). Initially identified as a binding partner of the Orc1 protein (lizuka and Stillman, 1999), Hbo1 binds to Mcm2 and is required for pre-RC formation (Fig. 3) (lizuka et al., 2006). $\mathrm{Hbo1}$, the enzyme responsible for histone $\mathrm{H} 4$ acetylation, is a subunit of a protein complex comprised of JADE1/2/3 paralogs, hEaf6 and ING5 (Doyon et al., 2006). This complex
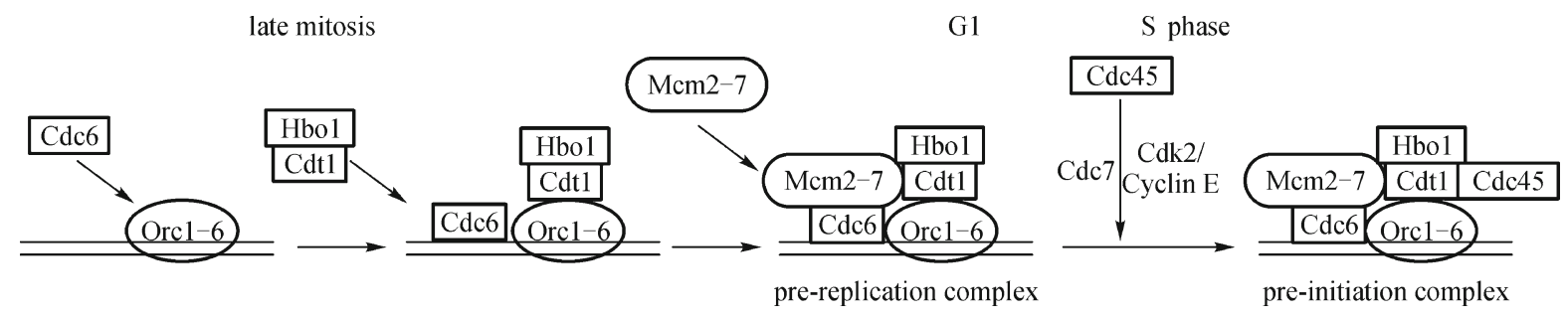

Figure 3. The order of protein loading onto replication origins to form pre-initiation complex. 
interacts with the $\mathrm{Mcm}$ helicase and is essential for DNA replication to occur during $S$ phase (Doyon et al., 2006). p53 tumor suppressor also associates with Hbo1 and ING5 in a shared protein complex and negatively regulates both $\mathrm{Hbo} 1$ HAT activity and Hbo1-mediated chromatin loading of $\mathrm{Mcm}$ proteins (lizuka et al., 2007). Recently, it was shown that $\mathrm{Hbo} 1$ acts as a coactivator of the licensing factor Cdt1 (Miotto and Struhl, 2008) and that the HAT activity of $\mathrm{Hbo} 1$ toward $\mathrm{H} 4$ at replication origins is essential for DNA replication licensing and is inhibited by Geminin, an inhibitor of Cdt1 (Miotto and Struhl, 2010).

In a search for Plk1-interacting proteins, we have identified Hbo1 as a Plk1 target (Wu and Liu, 2008). We showed that the interaction between Plk1 and $\mathrm{Hbo} 1$ is mitosis specific and that Plk1 phosphorylates Hbo1 on Ser57 in vitro. During mitosis, Cdk1 phosphorylates Hbo1 on Thr85/88 creating a docking site for Plk1 to be recruited. Significantly, overexpression of $\mathrm{Hbo} 1$ mutated at the Plk1 phosphorylation site (S57A) leads to cell cycle arrest in G1/S phase, inhibition of chromatin loading of the $\mathrm{Mcm}$ complex, and a reduced DNA replication rate. Similarly, $\mathrm{Hbo} 1$ depletion results in decreased DNA replication and failure of $\mathrm{Mcm}$ complex binding to chromatin, both of which can be partially rescued by ectopic expression of wild type Hbo1, but not Hbo1-S57A. These results suggest that Plk1 phosphorylation of $\mathrm{Hbo} 1$ is required for pre-RC formation and DNA replication licensing. These data provided direct evidence that Plk1 phosphorylation of $\mathrm{Hbo1}$ at late mitosis licenses DNA replication in the next round of $S$ phase.

\section{PLK1 IN CHROMOSOME DYNAMICS}

During cell division, chromosomes undergo dramatical structure changes to facilitate the equal distribution of duplicated DNA into two daughter cells. The condensation of chromatin fiber is the initiation step, followed by sister chromatid formation on bipolar spindle and segregation in mitosis. Cdc5, the polo kinase in budding yeast, directly regulates condensin (an evolutionarily conserved multisubunit ATPase to condense chromatin) through phosphorylationmediated activation of its DNA supercoiling activity (St-Pierre et al., 2009). In mammals, Plk1 coordinates with PICH (PIk1interacting checkpoint "helicase") to maintain prometaphase chromosome arm architecture, and $\mathrm{PICH}$ depletion leads to loss of Plk1 on the chromosome arm and premature release of Cohesin (the protein complex responsible for holding the sister chromatids together until anaphase) (Kurasawa and Yu-Lee, 2010).

\section{Topoisomerase lla (Topo Ila)}

Topo Ila plays a critical role in chromosome architecture, and it is required for condensation and separation of mitotic chromosomes. We identified Topo lla as a Plk1 substrate ( $\mathrm{Li}$ et al., 2008). Plk1 phosphorylation of topollalpha at serine 1337 and serine 1534 dramatically increases its decatenation activity, which is critical for sister-chromatid segregation. Overexpression of the Plk1 unphosphorylatable topoll mutant resulted in accumulation of catenated DNA due to reduced catalytic activity, and activated the ATM/R-dependent DNAdamage checkpoint. Finally, overexpression of an unphosphorylatable topolla mutant led to S phase arrest, suggesting that Plk1-associated phosphorylation of Topo lla first occurs in S phase. This is another example that Plk1 can have interphase functions.

\section{Telomeric-repeat binding factor (Trf1)}

Trf1 is a protein with dual functions: cell cycle regulation and telomere control. Trf1 is involved in normal mitotic progression and the G2/M DNA damage checkpoint. For example, Trf1 has been found to localize to the mitotic spindle (Nakamura et al., 2001); interact with the microtubule regulator EB1; to promote microtubule polymerization in vitro (Nakamura et al., 2002). Furthermore, it was shown that ATM phosphorylates Ser219 of Trf1 in response to DNA double-strand breaks and ATM-mediated phosphorylation contributes to the ability of overexpressed Trf1 to induce arrest or apoptosis in G2/M phase in some settings (Kishi et al., 2001). A Trf1 mutant refractory to ATM phosphorylation on Ser219 potently induces mitotic entry and apoptosis and increases radiation hypersensitivity of ataxia-telangiectasia (A-T) disease cells. In contrast, Trf1 mutants mimicking ATM phosphorylation on Ser219 completely fail to induce apoptosis and also reduce radiation hypersensitivity of $\mathrm{A}-\mathrm{T}$ disease cells (Kishi et al., 2001).

In addition to the roles in mitosis, Trf1 also functions as a negative regulator of telomere maintenance, since expression of a dominant-negative mutant results in elongation of telomeres, and overexpression of WT Trf1 shortens telomeres (van Steensel and de Lange, 1997). Maintained by telomerase, telomeres are comprised of TTAGGG repeat arrays and two associated binding factors, Trf1 and Trf2. Although Trf1 has no effect on telomerase activity per se, Trf1 recruits a number of other proteins including TIN2 and tankyrase 1 to telomeres to regulate telomere length (Fig. 4A and 4B) (Kim et al., 1999; Smith and de Lange, 2000). How does the Trf1 complex, which is bound to the duplex telomeric DNA, regulate telomerase that acts at the $3^{\prime}$ overhang at a considerable distance? POT1 protein provides a solution. In addition to a single-stranded DNA binding domain in its $\mathrm{N}$ terminus, POT1 can also be recruited to the telomeric chromatin by the Trf1 complex, acting as a "proteincounting" device to measure telomere length (Loayza and De Lange, 2003). It was shown that POT1 functions downstream of the Trf1 complex to relay the negative regulation to the telomere terminus (Loayza and De Lange, 2003). Thus, as telomeres get longer, more Trf1 complex is present at the 
chromosome end, increasing the chance of POT1 being present on the single-stranded telomeric DNA where it would preclude telomerase from accessing the end (Smogorzewska and de Lange, 2004). Two possible mechanisms exist for POT1 inhibition of telomerase. Since POT1 has some preference to bind to its recognition site at a $3^{\prime}$ end, its physical presence there may simply block telomerase from accessing the end (Fig. 4A) (Loayza et al., 2004). A second model is based on the unusual architecture of telomeres (Fig. 4B). In mammals, the Trf2 complex promotes T-loop formation in which the $3^{\prime}$ telomeric overhang is invaded into the preceding duplex telomeric repeat array (Griffith et al., 1999). Because the single-stranded $3^{\prime}$ overhang of the telomere terminus is base-paired to the C-strand sequence in the Tloop configuration and telomerase requires an unpaired $3^{\prime}$ end (Lingner and Cech, 1996), the telomere terminus is unlikely to be accessible to telomerase. Due to its intrinsic ability to bind to single-stranded DNA (Baumann and Cech, 2001), POT1 should have the ability to bind to the displaced TTAGGG repeats at the base of the T-loop. The binding of POT1 could potentially stabilize T-loops (e.g., by preventing branch-migration) and thereby block telomerase from gaining access to the $3^{\prime}$ telomere terminus (Fig. 4B).

Although the role of Trf1 in telomere length control has been documented, increasing evidence suggests that Trf1 also play important roles for functional telomere structure. Using a conditional knockout approach, murine Trf1 has been shown to be essential for cell proliferation and chromosome stability (Iwano et al., 2004). Consistent with this, TIN2 binds Trf1 and Trf2 simultaneously and stabilizes the Trf2 complex on telomeres (Ye et al., 2004). Interestingly, a recent report showed that ATM and the Mre11-Rad50-Nbs1 complex, which takes part in recombinational DNA repair, cell cycle checkpoint control and telomere maintenance, function as comediators of Trf1 in telomere length control even in the absence of DNA damage (Wu et al., 2007). It was proposed that MRN is required for Trf1 phosphorylation by ATM and that such phosphorylation results in the release of Trf1 from telomeres, promoting telomerase access to the ends of telomeres (Wu et al., 2007). This finding suggests that ATMmediated phosphorylation may coordinate the dual roles of Trf1 in telomere length control and cell cycle regulation.

During the search for Plk1-interaction proteins, we have identified Trf1 as a Plk1 target (Wu et al., 2008). We showed that Plk1 forms a complex with Trf1, and Plk1 phosphorylates Trf1 at Ser435 in vivo. Moreover, Cdk1, serving as a priming kinase, phosphorylates Trf1 to generate a docking site for Plk1 toward Trf1. In the presence of nocodazole, a spindle poison to arrest cells at mitosis, ectopic expression of wild type Trf1 but not Trf1 with alanine mutation in the Plk1 phosphorylation site induced apoptosis in cells containing short telomeres, but not in cells containing long telomeres. Unexpectedly, downregulation of Trf1 by RNA interference affects cell proliferation and results in obvious apoptosis in cells with short telomeres, but not in cells with long telomeres. Importantly, we observed that telomeric DNA binding ability of Trf1 is cell cycle regulated and reaches a peak during mitosis. Upon phosphorylation by Plk1 in vivo and in vitro, Trf1's ability to bind telomeric DNA is dramatically increased. These results demonstrated that Plk1 interacts with and phosphorylates Trf1 and suggest that Plk1-mediated phosphorylation is involved in both Trf1 overexpression-induced apoptosis and
A

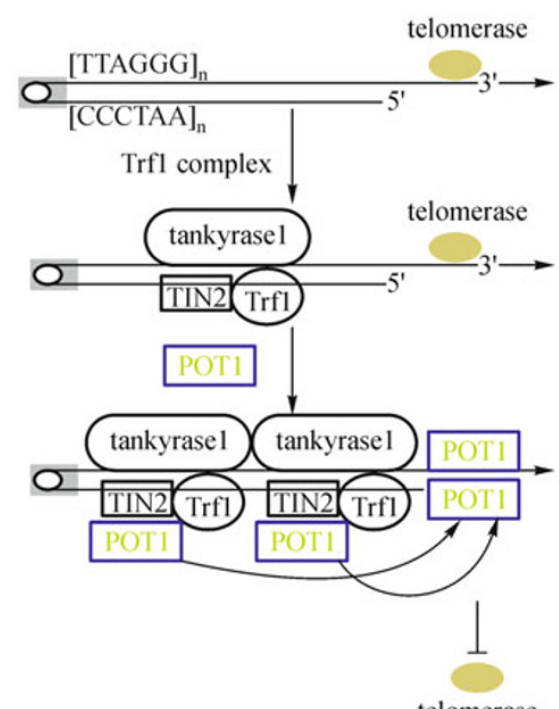

B

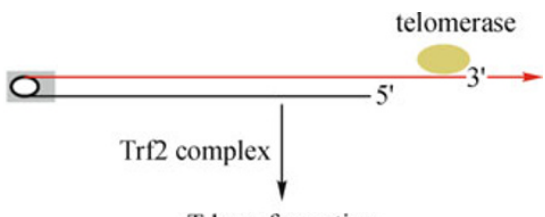

T-loop formation

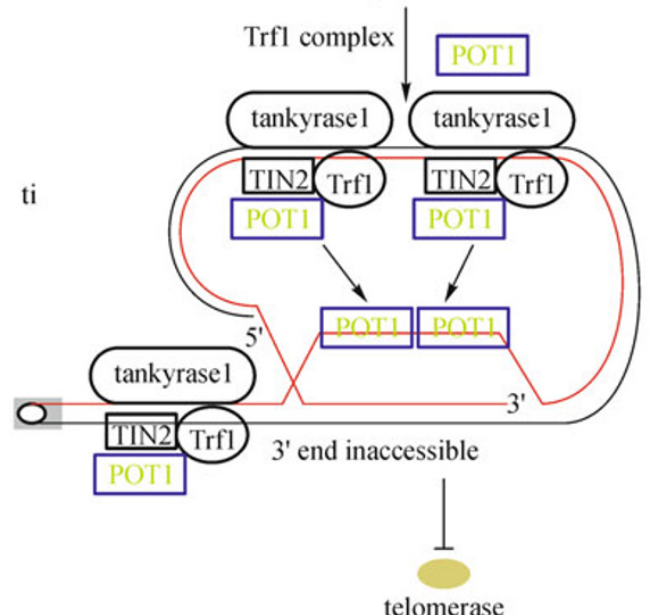

Figure 4. Two models of telomere length regulation by the Trf1 complex. 
its telomeric DNA binding ability. In other words, dual functions of Trf1, cell cycle regulation and telomere length control, are regulated by Plk1-associated kinase activity.

\section{PLK1 IN CHECKPOINT RECOVERY}

During cell-cycle progression, checkpoints function as the control mechanism to ensure the fidelity of cell division. The necessary processes for each cell-cycle phase are monitored by checkpoint network, and a cell cannot proceed to the next phase until checkpoint requirements have been met. Multiple checkpoints exist in the cell cycle, and DNA-damage checkpoint is one major type to ensure genomic stability. Accumulating evidence suggests that Plk1 plays a central role in recovery from the G2 DNA-damage checkpoint and is not dispensable for the onset of mitosis after DNA-damage signal is silenced (van Vugt et al., 2004). DNA double-strand breaks occurred in $\mathrm{G} 2$ phase are monitored by the Mre11Rad50-Nbs1 (MRN) complex at the local sites, and this complex further recruits the checkpoint kinases ATM (Ataxia telangiectasia mutated) and ATR (ataxia telangiectasia and Rad3-related protein) to amplify the DNA damage signal through several downstream targets (Bartek and Lukas, 2007). CHK2 and CHK1 are the major effector kinases for ATM and ATR respectively, and they lead to G2 cell cycle arrest by inhibiting the Cdc25 family of phosphatase to prevent Cdc25 family members from activating cyclin-Cdk complexes (Sanchez et al., 1997; Lam and Rosen, 2004). The tumor suppressor p53 is also phosphorylated and activated by CHK $1 / 2$ and transcriptionally upregulates $p 21$, the universal inhibitor of Cdk, to maintain the $\mathrm{G} 2$ checkpoint stage (Bunz et al., 1998). This will allow cells to have enough time for efficient DNA repair. During the subsequent recovery process from the G2 DNA damage-induced arrest, ATM/RCHK2/1-p53 checkpoint network needs to be silenced and thus cyclin-Cdk can be re-activated. During this process, Plk1 is first activated by Aurora A kinase via the phosphorylation at its activation loop (threonine 210), and its kinase activity is critical for cyclin B-Cdk1 reactivation for mitotic entry (Macůrek et al., 2008). Depletion of Plk1 by RNAi leads to constitutive activation of checkpoint signal and recovery failure. Later, Plk1 was found to target several essential components in the checkpoint network to silence the checkpoint signal.

Claspin, an adaptor protein for ATR to activate CHK1 during the replicative stress, was the first Plk1 substrate that is involved in regulating checkpoint recovery (Mamely et al., 2006; Peschiaroli et al., 2006). Plk1-mediated phosphorylation in a canonical DSGxxS degron sequence of Claspin recruits the ubiquitin ligase $\beta$-TrCP to trigger ubiquitinationdependent degradation of Claspin in $\mathrm{G} 2$ phase. This degradation event is necessary for the termination of DNA replication checkpoint, and thus allows cells enter into mitosis. In parallel with ATR-Claspin-CHK1 pathway, ATM-
53BP1-CHK2 pathway was also regulated by Plk1 (van Vugt et al., 2010). Besides involved in NHEJ DNA repair network, 53BP1 interacts with a range of effector checkpoint proteins including CHK2 and p53 at the DNA damage foci. During the G2 checkpoint recovery, Plk1 is recruited by 53BP1 through the interaction between Polo-box domain of Plk1 and the docking site of 53BP1 generated by Cdk1. Upon recruitment, Plk1 directly phosphorylates CHK2 at its ForkheadAssociated (FHA) domain and inactivate CHK2 kinase, thus silencing the ATM-53BP1-CHK2 pathway.

Forkhead transcription factor FoxM1, one of the major transcriptional regulators for $\mathrm{G} 2 / \mathrm{M}$ transition, was also identified as a Plk1 substrate (Fu et al., 2008). In this case, Plk1 phosphorylation functions in a feed-forward manner to further promote mitosis-specific gene expression. Later, FoxM1 was found to be essential to maintain the recovery competence during the G2 DNA damage-induced arrest (AlvarezFernández et al., 2010). Although Cdk1-dependent phosphorylation of FoxM1 was shown to be critical for this maintenance, it is reasonable to speculate that Plk1associated kinase activity toward FoxM1 might ensure enough accumulation of mitotic gene expression needed for recovery from the G2 DNA-damage checkpoint.

\section{PLK1 IN p53 REGULATION}

One striking phenotype we initially reported is stabilization of p53 tumor suppressor in Plk1-depleted HeLa cells (Liu and Erikson, 2003). A later study showed that Plk1 indeed physically binds to the DNA binding domain of p53 in cultured cells. Moreover, expression of exogenous Plk1 and tumor suppressor p53 in p53 null H1299 cells greatly decreased p53-mediated transcription from the p53-responsive p21, MDM2, and Bax promoters, whereas kinase-deficient Plk1 failed to reduce the transcriptional activity of p53 (Ando et al., 2004). These data suggest that Plk1-mediated negative regulation of p53 might be a fundamental mechanism for the role of Plk1 in oncogenesis. Although it was reported that Plk1 directly phosphorylates p53 in vitro (Xie et al., 2001), we have not been able to repeat this result. We have extensive experience with Plk1 substrates, and feel confident that p53 is not a Plk1 substrate in vivo. In a search for Plk1-interacting partners, we have identified two potential Plk1 substrates: Topors (topoisomerase I binding protein) and GTSE1 (G2 and $S$ phase-expressed protein 1). Interestingly, both Topors and GTSE1 have established functions in p53 regulation.

\section{Topors}

Topors is a protein that was originally discovered in a screen searching for proteins that bind to DNA topoisomerase I (Haluska et al., 1999), and was also identified as a p53 binding protein (Zhou et al., 1999). Although Topors is widely expressed in normal human tissues, its expression is 
decreased or undetectable in colon, lung and brain adenocarcinomas, indicating that it might function as a tumor suppressor (Saleem et al., 2004). Consistent with this concept, the human Topors gene is located on chromosome 9p21 (Chu et al., 2001); loss of heterozygosity in this region is frequently observed in several different malignancies. Forced expression of Topors in cancer cells inhibits cellular proliferation and colony formation, likely due to G0/G1 arrest and apoptosis (Saleem et al., 2004; Lin et al., 2005). Topors associates with promyelocytic leukemia (PML) nuclear bodies in the nuclei of exponentially growing cells. Treatment with transcriptional inhibitors or with the topoisomerase l-targeting drug camptothecin causes rapid dispersion of Topors to the nucleoplasm, indicating that Topors may be involved in the cellular response to camptothecin (Rasheed et al., 2002). Topors contains an N-terminal C3HC4-type RING domain that is closely related in sequence to the RING domains of known E3 ligases (Fig. 5A), and is the first example of a protein with both ubiquitin and SUMO-1 E3 ligase activity. Topors functions as an E3 ubiquitin ligase for the transcription factor p53, Hairy and NKX3.1, and Topors-dependent ubiquitination leads to the degradation of these proteins (Rajendra et al., 2004; Guan et al., 2008). Substrates of the SUMO-1 E3 ligase activity of Topors include DNA topoisomerase I, many chromatin-modifying proteins and p53 (Weger et al., 2005; Hammer et al., 2007). In contrast to ubiquitinationinduced protein degradation, Topors-induced p53 sumoylation is accompanied by an increase in p53 protein levels in HeLa cells (Weger et al., 2005). Therefore, it is possible that the effects of Topors on a particular substrate, such as p53, may vary depending on the cellular context and the level of Topors (Guan et al., 2008). We recently identified Topors as a Plk1 target (Yang et al., 2009b). We showed that Plk1 phosphorylates Topors on Ser718 in vivo. Significantly, expression of a Plk1-unphosphorylatable Topors mutant (S718A) leads to a dramatic accumulation of p53, through inhibition of p53 degradation. Mechanistically, Plk1-mediated phosphorylation of Topors inhibits Topors-mediated sumoylation of p53, while p53 ubiquitination is enhanced, leading to p53 degradation (Fig. 5D). These results demonstrated that Plk1 modulates Topors activity in suppressing p53 function, and identify a likely mechanism for the tumorigenic potential of Plk1.

\section{GTSE1}

Originally isolated during a screening of $\mathrm{p} 53$-inducible genes, GTSE1 (G2 and $S$ phase-expressed 1) is specifically expressed during $S$ and $G 2$ phases of the cell cycle. It is localized mainly to the microtubules and, when overexpressed, delays the $\mathrm{G} 2$ to $M$ transition (Utrera et al., 1998). In response to DNA damage, GTSE1 accumulates in the nucleus and binds to p53 directly through its C-terminal domain (Fig. 5B), consequently shuttling p53 out of the nucleus and repressing its ability to induce apoptosis (Monte et al., 2003; Monte et al., 2004). Therefore, it was proposed that after DNA damage, GTSE1 could play a dual role during the $\mathrm{G} 2$ checkpoint, promoting delay of the $\mathrm{G} 2$ to $\mathrm{M}$ transition and, at the same time, protecting these cells from p53dependent apoptosis (Monte et al., 2003). We have identified
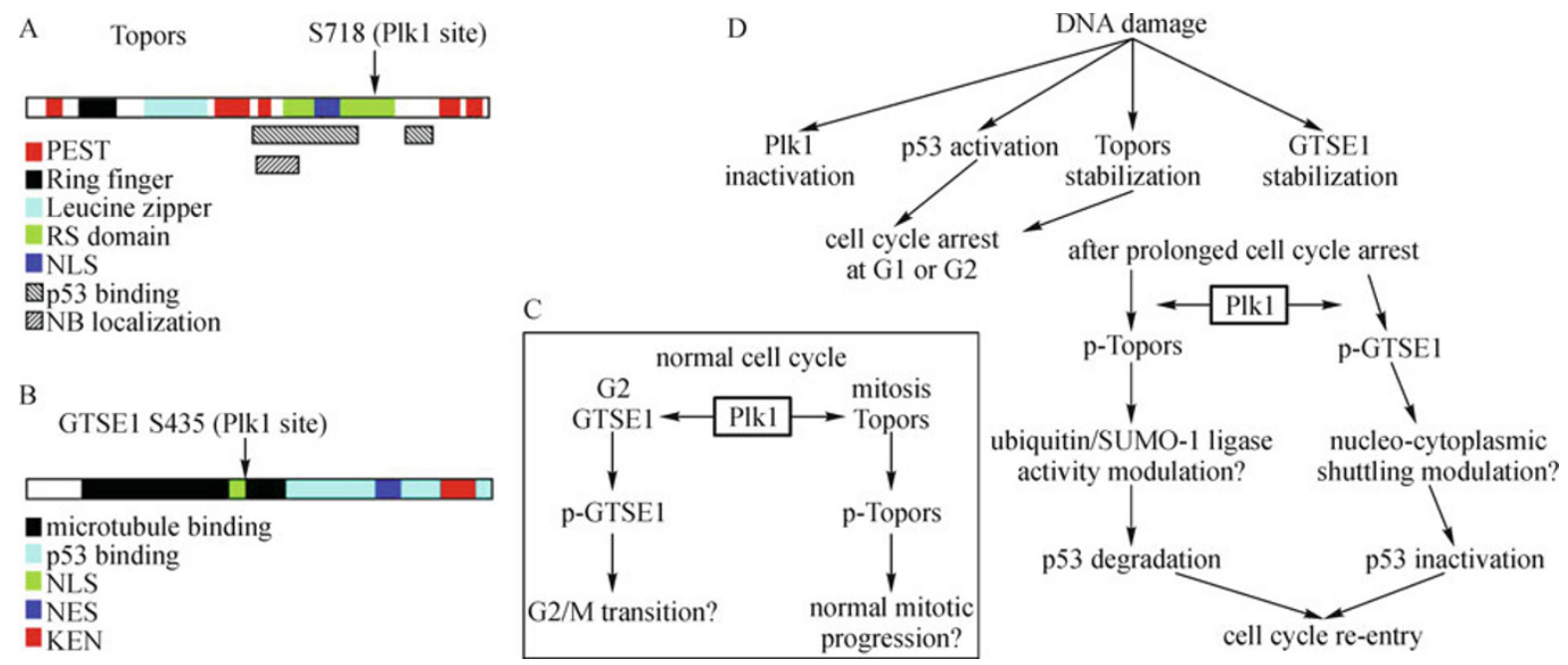

Figure 5. Proposed working models how Plk1 regulates p53. (A) Schematic representation of the domain structure of Topors. Two separate regions encoding putative p53 binding domains are aa 456-731 and 854-916. Amino-acid residues in the putative Ring finger motif and bipartite NLS are shown in black and blue boxes, respectively. PEST: sequences rich in Pro, Glu, Ser and Thr; RS domain: Arg and Ser rich; NLS: nuclear localization signal; NB: nuclear bodies. (B) Schematic representation of the GTSE1 domain structure. The putative microtubule binding domain (aa 150-378) and p53 binding domain (aa 476-720) are indicated by black and cyan boxes, respectively. NES: nuclear export signal. (C) Possible roles of Plk1 phosphorylation of GTSE1 and Topors in normal cell cycle progression. (D) Possible roles of Plk1 phosphorylation of GTSE1 and Topors in DNA-damage recovery. 
GTSE1 as another PIk1 substrate during the G2 checkpoint recovery (Liu et al., 2010). We showed that Plk1 phosphorylates GTSE1 at serine 435, and this phosphorylation leads to exposure of the nuclear-import signal of GTSE1 to the cellular transport machinery. As a result of the phosphorylation event, GTSE1 is able to translocate into the nucleus, bind with p53, and consequently shuttle it out of the nucleus. In the cytoplasm, p53 is rapidly degraded by the proteasome machinery. Without p53-mediated upregulation of p21 in the nucleus, this short half-life protein is quickly turned over, and cells can re-enter mitosis (Fig. 5D).

Because both Plk1 activity and p53 inactivation are required for exit from the G2 DNA-damage checkpoint, we hypothesize that Plk1 phosphorylation of Topors and GTSE1 leads to inactivation of the p53 pathway during recovery from the G2 DNA-damage checkpoint. Our current working models are as follows: During the normal cell cycle, Plk1 phosphorylation of GTSE1 and Topors might regulate their functions in the G2/M transition and mitotic progression, respectively (Fig. $5 \mathrm{C})$. In the presence of DNA damage, Plk1 is inactivated while the p53 pathway is turned on, to result in cell cycle arrest. Both Topors and GTSE1 levels are increased in response to DNA damage, and Topors might positively contribute to cell cycle arrest (Fig. 5D). During subsequent recovery from the G2 DNA-damage checkpoint after a prolonged cell cycle arrest, Plk1 is phosphorylated at T210 by Bora/aurora A and activated. Activated PIk1 subsequently phosphorylates both Topors and GTSE1. Whereas PIk1 phosphorylation of Topors might regulate its ubiquitin/ SUMO-1 ligase activity toward p53, resulting in p53 degradation, Plk1 phosphorylation of GTSE1 might regulate its nucleo/cytoplasmic shuttling, subsequently resulting in p53 inactivation. Both events will contribute to cell cycle reentry into mitosis (Fig. 5D).

\section{OUTLOOK AND FUTURE DIRECTION}

Ever since the discovery of Plk1 in mammalian cells, many important Plk1 substrates have been identified. Characterization of these substrates has given great insight in understanding the phosphorylation-mediated regulation, which plays a critical role in diverse cell cycle-related events. Recently, the mass spectrometry-based proteomics approach dramatically accelerates the substrate identification process. 622 proteins were identified as the interactome for Polo-box domain of Plk1 in Michael Yaffe's laboratory (Lowery et al., 2007). These potential Plk1 substrates have functions that are not limited to well established Plk1-regulated processes, such as cell-cycle regulation, but also are proteins with functions in diverse cellular events, including translational control, RNA processing, and vesicle transport. Although much more detailed biochemical and cellular characterizations are needed to validate these potential Plk1 substrates, it is almost certain that Plk1 has many functions beyond mitosis. In addition, the capacity of mass spectrometry- based proteomics allows us to study phosphorylation in the scope of cellular compartments, each of which contains hundreds of protein. Compared to individual characterization of each substrate, this method considers the fact that most simple biologic functions are mediated by protein network instead of certain single protein. One pioneering example of this approach is the study of Plk1-dependent phosphoproteome on the early mitotic spindle in Eric Nigg's laboratory (Santamaria et al., 2010). In this study, mitotic spindles were purified and 358 phosphorylation sites associated with mitotic spindles were identified. Importantly, 102 potential Plk1 phosphorylation sites were validated in vitro and most of them had not been reported before. Undoubtedly, this is an exciting time for Plk1 research.

\section{ACKNOWLEDGEMENTS}

We apologize to the scientists who made contributions to the field, but have not been cited due to space limitations.

\section{REFERENCES}

Aggarwal, B.D., and Calvi, B.R. (2004). Chromatin regulates origin activity in Drosophila follicle cells. Nature 430, 372-376.

Alvarez-Fernández, M., Halim, V.A., Krenning, L., Aprelia, M., Mohammed, S., Heck, A.J., and Medema, R.H. (2010). Recovery from a DNA-damage-induced $\mathrm{G} 2$ arrest requires Cdk-dependent activation of FoxM1. EMBO Rep 11, 452-458.

Ando, K., Ozaki, T., Yamamoto, H., Furuya, K., Hosoda, M., Hayashi, S., Fukuzawa, M., and Nakagawara, A. (2004). Polo-like kinase 1 (Plk1) inhibits p53 function by physical interaction and phosphorylation. J Biol Chem 279, 25549-25561.

Bartek, J., and Lukas, J. (2007). DNA damage checkpoints: from initiation to recovery or adaptation. Curr Opin Cell Biol 19, 238-245.

Baumann, C., Körner, R., Hofmann, K., and Nigg, E.A. (2007). PICH, a centromere-associated SNF2 family ATPase, is regulated by Plk1 and required for the spindle checkpoint. Cell 128, 101-114.

Baumann, P., and Cech, T.R. (2001). Pot1, the putative telomere endbinding protein in fission yeast and humans. Science 292, 1171-1175.

Brunner, D., and Nurse, P. (2000). CLIP170-like tip1p spatially organizes microtubular dynamics in fission yeast. Cell 102, 695-704.

Budde, P.P., Kumagai, A., Dunphy, W.G., and Heald, R. (2001). Regulation of Op18 during spindle assembly in Xenopus egg extracts. J Cell Biol 153, 149-158.

Bunz, F., Dutriaux, A., Lengauer, C., Waldman, T., Zhou, S., Brown, J. P., Sedivy, J.M., Kinzler, K.W., and Vogelstein, B. (1998). Requirement for p53 and p21 to sustain G2 arrest after DNA damage. Science 282, 1497-1501.

Casenghi, M., Meraldi, P., Weinhart, U., Duncan, P.I., Körner, R., and Nigg, E.A. (2003). Polo-like kinase 1 regulates NIp, a centrosome protein involved in microtubule nucleation. Dev Cell 5, 113-125.

Choi, J.H., Bertram, P.G., Drenan, R., Carvalho, J., Zhou, H.H., and Zheng, X.F. (2002). The FKBP12-rapamycin-associated protein (FRAP) is a CLIP-170 kinase. EMBO Rep 3, 988-994.

Chu, D., Kakazu, N., Gorrin-Rivas, M.J., Lu, H.P., Kawata, M., Abe, T., 
Ueda, K., and Adachi, Y. (2001). Cloning and characterization of LUN, a novel ring finger protein that is highly expressed in lung and specifically binds to a palindromic sequence. J Biol Chem 276, 14004-14013.

Coquelle, F.M., Caspi, M., Cordelières, F.P., Dompierre, J.P., Dujardin, D.L., Koifman, C., Martin, P., Hoogenraad, C.C., Akhmanova, A., Galjart, N., et al. (2002). LIS1, CLIP-170's key to the dynein/dynactin pathway. Mol Cell Biol 22, 3089-3102.

Dhar, S.K., Delmolino, L., and Dutta, A. (2001). Architecture of the human origin recognition complex. J Biol Chem 276, 29067-29071.

Doyon, Y., Cayrou, C., Ullah, M., Landry, A.J., Côté, V., Selleck, W., Lane, W.S., Tan, S., Yang, X.J., and Côté, J. (2006). ING tumor suppressor proteins are critical regulators of chromatin acetylation required for genome expression and perpetuation. Mol Cell 21, 51-64.

Eckerdt, F., Yuan, J., and Strebhardt, K. (2005). Polo-like kinases and oncogenesis. Oncogene 24, 267-276.

Elia, A.E., Cantley, L.C., and Yaffe, M.B. (2003). Proteomic screen finds $\mathrm{pSer} / \mathrm{pThr}$-binding domain localizing Plk1 to mitotic substrates. Science 299, 1228-1231.

Elowe, S., Hümmer, S., Uldschmid, A., Li, X., and Nigg, E.A. (2007). Tension-sensitive Plk1 phosphorylation on BubR1 regulates the stability of kinetochore microtubule interactions. Genes Dev 21, 2205-2219.

Fu, Z., Malureanu, L., Huang, J., Wang, W., Li, H., van Deursen, J.M., Tindall, D.J., and Chen, J. (2008). Plk1-dependent phosphorylation of FoxM1 regulates a transcriptional programme required for mitotic progression. Nat Cell Biol 10, 1076-1082.

Fukata, M., Watanabe, T., Noritake, J., Nakagawa, M., Yamaga, M., Kuroda, S., Matsuura, Y., Iwamatsu, A., Perez, F., and Kaibuchi, K. (2002). Rac1 and Cdc42 capture microtubules through IQGAP1 and CLIP-170. Cell 109, 873-885.

Griffith, J.D., Comeau, L., Rosenfield, S., Stansel, R.M., Bianchi, A., Moss, H., and de Lange, T. (1999). Mammalian telomeres end in a large duplex loop. Cell 97, 503-514.

Guan, B., Pungaliya, P., Li, X., Uquillas, C., Mutton, L.N., Rubin, E.H., and Bieberich, C.J. (2008). Ubiquitination by TOPORS regulates the prostate tumor suppressor NKX3.1. J Biol Chem 283, 4834-4840.

Haluska, P. Jr, Saleem, A., Rasheed, Z., Ahmed, F., Su, E.W., Liu, L. F., and Rubin, E.H. (1999). Interaction between human topoisomerase I and a novel RING finger/arginine-serine protein. Nucleic Acids Res 27, 2538-2544.

Hammer, E., Heilbronn, R., and Weger, S. (2007). The E3 ligase Topors induces the accumulation of polysumoylated forms of DNA topoisomerase I in vitro and in vivo. FEBS Lett 581, 5418-5424.

lizuka, M., Matsui, T., Takisawa, H., and Smith, M.M. (2006). Regulation of replication licensing by acetyltransferase $\mathrm{Hbo} 1$. Mol Cell Biol 26, 1098-1108.

lizuka, M., Sarmento, O.F., Sekiya, T., Scrable, H., Allis, C.D., and Smith, M.M. (2007). Hbo1 Links p53-Dependent Stress Signaling to DNA Replication Licensing. Mol Cell Biol.

lizuka, M., and Stillman, B. (1999). Histone acetyltransferase HBO1 interacts with the ORC1 subunit of the human initiator protein. $J$ Biol Chem 274, 23027-23034.

Iwano, T., Tachibana, M., Reth, M., and Shinkai, Y. (2004). Importance of TRF1 for functional telomere structure. J Biol Chem 279,
1442-1448.

Kang, Y.H., Park, J.E., Yu, L.R., Soung, N.K., Yun, S.M., Bang, J.K., Seong, Y.S., Yu, H., Garfield, S., Veenstra, T.D., et al. (2006). Selfregulated Plk1 recruitment to kinetochores by the Plk1-PBIP1 interaction is critical for proper chromosome segregation. Mol Cell 24, 409-422.

Kim, S.H., Kaminker, P., and Campisi, J. (1999). TIN2, a new regulator of telomere length in human cells. Nat Genet 23, $405-412$.

Kishi, S., Zhou, X.Z., Ziv, Y., Khoo, C., Hill, D.E., Shiloh, Y., and Lu, K. P. (2001). Telomeric protein Pin2/TRF1 as an important ATM target in response to double strand DNA breaks. J Biol Chem 276, 29282-29291.

Knecht, R., Elez, R., Oechler, M., Solbach, C., von Ilberg, C., and Strebhardt, K. (1999). Prognostic significance of polo-like kinase (PLK) expression in squamous cell carcinomas of the head and neck. Cancer Res 59, 2794-2797.

Knecht, R., Oberhauser, C., and Strebhardt, K. (2000). PLK (polo-like kinase), a new prognostic marker for oropharyngeal carcinomas. Int J Cancer 89, 535-536.

Komarova, Y.A., Akhmanova, A.S., Kojima, S., Galjart, N., and Borisy, G.G. (2002). Cytoplasmic linker proteins promote microtubule rescue in vivo. J Cell Biol 159, 589-599.

Kurasawa, Y., and Yu-Lee, L.Y. (2010). PICH and cotargeted Plk1 coordinately maintain prometaphase chromosome arm architecture. Mol Biol Cell 21, 1188-1199.

Lam, M.H., and Rosen, J.M. (2004). Chk1 versus Cdc25: chking one's levels of cellular proliferation. Cell Cycle 3, 1355-1357.

Lansbergen, G., Komarova, Y., Modesti, M., Wyman, C., Hoogenraad, C.C., Goodson, H.V., Lemaitre, R.P., Drechsel, D.N., van Munster, E., Gadella, T.W. Jr, et al. (2004). Conformational changes in CLIP-170 regulate its binding to microtubules and dynactin localization. J Cell Biol 166, 1003-1014.

Li, H., Liu, X.S., Yang, X., Song, B., Wang, Y., and Liu, X. (2010b). Polo-like kinase 1 phosphorylation of $p 150 G$ lued facilitates nuclear envelope breakdown during prophase. Proc Natl Acad Sci U S A 107, 14633-14638.

Li, H., Liu, X.S., Yang, X., Wang, Y., Wang, Y., Turner, J.R., and Liu, X. (2010a). Phosphorylation of CLIP-170 by Plk1 and CK2 promotes timely formation of kinetochore-microtubule attachments. EMBO J 29, 2953-2965.

Li, H., Wang, Y., and Liu, X. (2008). Plk1-dependent phosphorylation regulates functions of DNA topoisomerase Ilalpha in cell cycle progression. J Biol Chem 283, 6209-6221.

Liang, C., Weinreich, M., and Stillman, B. (1995). ORC and Cdc6p interact and determine the frequency of initiation of DNA replication in the genome. Cell 81, 667-676.

Lin, L., Ozaki, T., Takada, Y., Kageyama, H., Nakamura, Y., Hata, A., Zhang, J.H., Simonds, W.F., Nakagawara, A., and Koseki, H. (2005). topors, a p53 and topoisomerase I-binding RING finger protein, is a coactivator of p53 in growth suppression induced by DNA damage. Oncogene 24, 3385-3396.

Lingner, J., and Cech, T.R. (1996). Purification of telomerase from Euplotes aediculatus: requirement of a primer $3^{\prime}$ overhang. Proc Natl Acad Sci U S A 93, 10712-10717.

Liu, X., and Erikson, R.L. (2003). Polo-like kinase (PIk)1 depletion induces apoptosis in cancer cells. Proc Natl Acad Sci U S A 100, 5789-5794. 
Liu, X., Lin, C.Y., Lei, M., Yan, S., Zhou, T., and Erikson, R.L. (2005). CCT chaperonin complex is required for the biogenesis of functional Plk1. Mol Cell Biol 25, 4993-5010.

Liu, X.S., Li, H., Song, B., and Liu, X. (2010). Polo-like kinase 1 phosphorylation of $\mathrm{G} 2$ and $\mathrm{S}$-phase-expressed 1 protein is essential for p53 inactivation during $\mathrm{G} 2$ checkpoint recovery. EMBO Rep 11, 626-632.

Llamazares, S., Moreira, A., Tavares, A., Girdham, C., Spruce, B.A., Gonzalez, C., Karess, R.E., Glover, D.M., and Sunkel, C.E. (1991). polo encodes a protein kinase homolog required for mitosis in Drosophila. Genes Dev 5, 2153-2165.

Loayza, D., and De Lange, T. (2003). POT1 as a terminal transducer of TRF1 telomere length control. Nature 423, 1013-1018.

Loayza, D., Parsons, H., Donigian, J., Hoke, K., and de Lange, T. (2004). DNA binding features of human POT1: a nonamer 5'TAGGGTTAG-3' minimal binding site, sequence specificity, and internal binding to multimeric sites. J Biol Chem 279, 13241-13248.

Lowery, D.M., Clauser, K.R., Hjerrild, M., Lim, D., Alexander, J., Kishi, K., Ong, S.E., Gammeltoft, S., Carr, S.A., and Yaffe, M.B. (2007). Proteomic screen defines the Polo-box domain interactome and identifies Rock2 as a Plk1 substrate. EMBO J 26, 2262-2273.

Macůrek, L., Lindqvist, A., Lim, D., Lampson, M.A., Klompmaker, R., Freire, R., Clouin, C., Taylor, S.S., Yaffe, M.B., and Medema, R.H. (2008). Polo-like kinase-1 is activated by aurora A to promote checkpoint recovery. Nature 455, 119-123.

Mamely, I., van Vugt, M.A., Smits, V.A., Semple, J.I., Lemmens, B., Perrakis, A., Medema, R.H., and Freire, R. (2006). Polo-like kinase-1 controls proteasome-dependent degradation of Claspin during checkpoint recovery. Curr Biol 16, 1950-1955.

Miotto, B., and Struhl, K. (2008). HBO1 histone acetylase is a coactivator of the replication licensing factor Cdt1. Genes Dev 22, 2633-2638.

Miotto, B., and Struhl, K. (2010). HBO1 histone acetylase activity is essential for DNA replication licensing and inhibited by Geminin. Mol Cell 37, 57-66.

Mitchison, T., and Kirschner, M. (1984). Dynamic instability of microtubule growth. Nature 312, 237-242.

Monte, M., Benetti, R., Buscemi, G., Sandy, P., Del Sal, G., and Schneider, C. (2003). The cell cycle-regulated protein human GTSE-1 controls DNA damage-induced apoptosis by affecting p53 function. J Biol Chem 278, 30356-30364.

Monte, M., Benetti, R., Collavin, L., Marchionni, L., Del Sal, G., and Schneider, C. (2004). hGTSE-1 expression stimulates cytoplasmic localization of p53. J Biol Chem 279, 11744-11752.

Nakamura, M., Zhou, X.Z., Kishi, S., Kosugi, I., Tsutsui, Y., and Lu, K. P. (2001). A specific interaction between the telomeric protein Pin2/ TRF1 and the mitotic spindle. Curr Biol 11, 1512-1516.

Nakamura, M., Zhou, X.Z., Kishi, S., and Lu, K.P. (2002). Involvement of the telomeric protein Pin2/TRF1 in the regulation of the mitotic spindle. FEBS Lett 514, 193-198.

Pacek, M., Tutter, A.V., Kubota, Y., Takisawa, H., and Walter, J.C. (2006). Localization of MCM2-7, Cdc45, and GINS to the site of DNA unwinding during eukaryotic DNA replication. Mol Cell 21, 581-587.

Perez, F., Diamantopoulos, G.S., Stalder, R., and Kreis, T.E. (1999). CLIP-170 highlights growing microtubule ends in vivo. Cell 96, 517-527.
Peschiaroli, A., Dorrello, N.V., Guardavaccaro, D., Venere, M., Halazonetis, T., Sherman, N.E., and Pagano, M. (2006). SCFbetaTrCP-mediated degradation of Claspin regulates recovery from the DNA replication checkpoint response. Mol Cell 23, 319-329.

Rajendra, R., Malegaonkar, D., Pungaliya, P., Marshall, H., Rasheed, Z., Brownell, J., Liu, L.F., Lutzker, S., Saleem, A., and Rubin, E.H. (2004). Topors functions as an E3 ubiquitin ligase with specific E2 enzymes and ubiquitinates p53. J Biol Chem 279, 36440-36444.

Rasheed, Z.A., Saleem, A., Ravee, Y., Pandolfi, P.P., and Rubin, E.H. (2002). The topoisomerase I-binding RING protein, topors, is associated with promyelocytic leukemia nuclear bodies. Exp Cell Res 277, 152-160.

Rickard, J.E., and Kreis, T.E. (1991). Binding of pp170 to microtubules is regulated by phosphorylation. J Biol Chem 266, 17597-17605.

Saleem, A., Dutta, J., Malegaonkar, D., Rasheed, F., Rasheed, Z., Rajendra, R., Marshall, H., Luo, M., Li, H., and Rubin, E.H. (2004). The topoisomerase I- and p53-binding protein topors is differentially expressed in normal and malignant human tissues and may function as a tumor suppressor. Oncogene 23, 5293-5300.

Sanchez, Y., Wong, C., Thoma, R.S., Richman, R., Wu, Z., PiwnicaWorms, H., and Elledge, S.J. (1997). Conservation of the Chk1 checkpoint pathway in mammals: linkage of DNA damage to Cdk regulation through Cdc25. Science 277, 1497-1501.

Santamaria, A., Wang, B., Elowe, S., Malik, R., Zhang, F., Bauer, M., Schmidt, A., Sillje, H.H., Koerner, R., and Nigg, E.A. (2010). The Plk1-dependent phosphoproteome of the early mitotic spindle. Mol Cell Proteomics. In press.

Smith, M.R., Wilson, M.L., Hamanaka, R., Chase, D., Kung, H., Longo, D.L., and Ferris, D.K. (1997). Malignant transformation of mammalian cells initiated by constitutive expression of the pololike kinase. Biochem Biophys Res Commun 234, 397-405.

Smith, S., and de Lange, T. (2000). Tankyrase promotes telomere elongation in human cells. Curr Biol 10, 1299-1302.

Smogorzewska, A., and de Lange, T. (2004). Regulation of telomerase by telomeric proteins. Annu Rev Biochem 73, 177208.

Spänkuch, B., Matthess, Y., Knecht, R., Zimmer, B., Kaufmann, M., and Strebhardt, K. (2004). Cancer inhibition in nude mice after systemic application of U6 promoter-driven short hairpin RNAs against PLK1. J Natl Cancer Inst 96, 862-872.

St-Pierre, J., Douziech, M., Bazile, F., Pascariu, M., Bonneil, E., Sauvé, V., Ratsima, H., and D'Amours, D. (2009). Polo kinase regulates mitotic chromosome condensation by hyperactivation of condensin DNA supercoiling activity. Mol Cell 34, 416-426.

Strebhardt, K. (2010). Multifaceted polo-like kinases: drug targets and antitargets for cancer therapy. Nat Rev Drug Discov 9, 643-660.

Strebhardt, K., Kneisel, L., Linhart, C., Bernd, A., and Kaufmann, R. (2000). Prognostic value of pololike kinase expression in melanomas. JAMA 283, 479-480.

Stuermer, A., Hoehn, K., Faul, T., Auth, T., Brand, N., Kneissl, M., Pütter, V., and Grummt, F. (2007). Mouse pre-replicative complex proteins colocalise and interact with the centrosome. Eur J Cell Biol $86,37-50$.

Sunkel, C.E., and Glover, D.M. (1988). polo, a mitotic mutant of Drosophila displaying abnormal spindle poles. J Cell Sci 89 , 25-38.

Tai, C.Y., Dujardin, D.L., Faulkner, N.E., and Vallee, R.B. (2002). Role 
of dynein, dynactin, and CLIP-170 interactions in LIS1 kinetochore function. J Cell Biol 156, 959-968.

Takai, N., Hamanaka, R., Yoshimatsu, J., and Miyakawa, I. (2005). Polo-like kinases (Plks) and cancer. Oncogene 24, 287-291.

Takeda, D.Y., and Dutta, A. (2005). DNA replication and progression through S phase. Oncogene 24, 2827-2843.

Tanenbaum, M.E., Galjart, N., van Vugt, M.A., and Medema, R.H. (2006). CLIP-170 facilitates the formation of kinetochore-microtubule attachments. EMBO J 25, 45-57.

Tsou, M.F., Wang, W.J., George, K.A., Uryu, K., Stearns, T., and Jallepalli, P.V. (2009). Polo kinase and separase regulate the mitotic licensing of centriole duplication in human cells. Dev Cell 17, 344-354.

Tsvetkov, L., and Stern, D.F. (2005). Interaction of chromatinassociated Plk1 and Mcm7. J Biol Chem 280, 11943-11947.

Utrera, R., Collavin, L., Lazarević, D., Delia, D., and Schneider, C. (1998). A novel p53-inducible gene coding for a microtubulelocalized protein with G2-phase-specific expression. EMBO J 17, 5015-5025.

van Steensel, B., and de Lange, T. (1997). Control of telomere length by the human telomeric protein TRF1. Nature 385, 740-743.

van Vugt, M.A., Brás, A., and Medema, R.H. (2004). Polo-like kinase1 controls recovery from a G2 DNA damage-induced arrest in mammalian cells. Mol Cell 15, 799-811.

van Vugt, M.A., Gardino, A.K., Linding, R., Ostheimer, G.J., Reinhardt, H.C., Ong, S.E., Tan, C.S., Miao, H., Keezer, S.M., Li, J., et al. (2010). A mitotic phosphorylation feedback network connects Cdk1, Plk1, 53BP1, and Chk2 to inactivate the $\mathrm{G}(2) / \mathrm{M}$ DNA damage checkpoint. PLoS Biol 8, e1000287.

Vaughan, P.S., Miura, P., Henderson, M., Byrne, B., and Vaughan, K. T. (2002). A role for regulated binding of $p 150$ (Glued) to microtubule plus ends in organelle transport. J Cell Biol 158, 305-319.

Weger, S., Hammer, E., and Heilbronn, R. (2005). Topors acts as a SUMO-1 E3 ligase for p53 in vitro and in vivo. FEBS Lett 579, 5007-5012.
Wolf, G., Elez, R., Doermer, A., Holtrich, U., Ackermann, H., Stutte, H. J., Altmannsberger, H.M., Rübsamen-Waigmann, H., and Strebhardt, K. (1997). Prognostic significance of polo-like kinase (PLK) expression in non-small cell lung cancer. Oncogene 14, 543549.

Wu, Y., Xiao, S., and Zhu, X.D. (2007). MRE11-RAD50-NBS1 and ATM function as co-mediators of TRF1 in telomere length control. Nat Struct Mol Biol 14, 832-840.

Wu, Z.Q., and Liu, X. (2008). Role for Plk1 phosphorylation of Hbo1 in regulation of replication licensing. Proc Natl Acad Sci U S A 105, 1919-1924.

Wu, Z.Q., Yang, X., Weber, G., and Liu, X. (2008). Plk1 phosphorylation of TRF1 is essential for its binding to telomeres. J Biol Chem 283, 25503-25513.

Xie, S., Wu, H., Wang, Q., Cogswell, J.P., Husain, I., Conn, C., Stambrook, P., Jhanwar-Uniyal, M., and Dai, W. (2001). Plk3 functionally links DNA damage to cell cycle arrest and apoptosis at least in part via the p53 pathway. J Biol Chem 276, $43305-43312$.

Yang, X., Li, H., Liu, X.S., Deng, A., and Liu, X. (2009a). Cdc2mediated phosphorylation of CLIP-170 is essential for its inhibition of centrosome reduplication. J Biol Chem 284, 28775-28782.

Yang, X., Li, H., Zhou, Z., Wang, W.H., Deng, A., Andrisani, O., and Liu, X. (2009b). Plk1-mediated phosphorylation of Topors regulates p53 stability. J Biol Chem 284, 18588-18592.

Yarm, F.R. (2002). Plk phosphorylation regulates the microtubulestabilizing protein TCTP. Mol Cell Biol 22, 6209-6221.

Ye, J.Z., Donigian, J.R., van Overbeek, M., Loayza, D., Luo, Y., Krutchinsky, A.N., Chait, B.T., and de Lange, T. (2004). TIN2 binds TRF1 and TRF2 simultaneously and stabilizes the TRF2 complex on telomeres. J Biol Chem 279, 47264-47271.

Yim, H., and Erikson, R.L. (2009). Polo-like kinase 1 depletion induces DNA damage in early $S$ prior to caspase activation. Mol Cell Biol 29, 2609-2621.

Zhou, R., Wen, H., and Ao, S.Z. (1999). Identification of a novel gene encoding a p53-associated protein. Gene 235, 93-101. 